\title{
Nonlinear gyrotropic vortex dynamics in ferromagnetic dots
}

\author{
Konstantin Y. Guslienko \\ Departamento de Física de Materiales. Universidad del Pais Vasco, 20018 San Sebastian. Spain \\ and IKERBASQUE. The Basque Foundation for Science, 48011 Bilbao, Spain \\ Rafael Hernández Heredero \\ EUTT Teleconnunicación. Universidad Politécnica de Madrial. 28031 Madrial. Spain \\ Oksana Chubykalo-Fesenko \\ Instituto de Ciencia de Materiales de Madrid. CSIC, Cantoblanco, 28049 Madrid, Spain \\ (Received 16 April 2010; revised manuscript received 16 June 2010; published 8 July 2010)
}

\begin{abstract}
The quasistationary and transient (nanosecond) regimes of nonlinear vortex dynamics in a soft magnetic dot driven by an oscillating external field are studied. We derive a nonlinear dynamical system of equations for the vortex core position and phase, assuming that the main source of nonlinearity comes from the magnetostatic energy. In the stationary regime, we demonstrate the occurrence of a fold-over bifurcation and calculate analytically the resonant nonlinear vortex frequencies as a function of the amplitude and frequency of the applied driving field. In the transient regime. we show that the vortex core dynamics are described by an oscillating trajectory radius. The resulting dynamics contain multiple frequencies with amplitude decaying in time. Finally. we evaluate the ranges of the system parameters leading to a vortex core instability (core polarization reversal).
\end{abstract}

DOI: $10.1103 /$ PhysRevB.82.014402

PACS number(s): 75.78.-n, 75.75.Jn, 76.90.+d

\section{INTRODUCTION}

Fast magnetization dynamics in geometrically confined magnetic elements on submicron length scales is currently attracting fundamental interest and it is crucially important for the future advancements of nanomagnetism and spintronics. Studying the spin-excitation eigenmodes in such systems provides valuable information to relate the nanoelement $d y-$ namical response to its geometrical and magnetic parameters relevant for spintronic circuits operating in the gigahertz frequency range. The closely related problem of the dynamics of magnetization reversal in patterned nanostructures is also of great relevance for applications. The understanding of magnetization dynamics in restricted geometries can be achieved by combining theoretical calculations, micromagnetic simulations, and experimental approaches. Broadband ferromagnetic resonance measurements on single magnetic submicron particles of nanoscale thickness using the strip line technique provide unique information on the dynamics in the frequency domain. ${ }^{1-3}$ Alternatively, time-resolved $\mathrm{Kerr}^{4-6}$ and $\mathrm{X}$-ray magnetic circular dichroism (XMCD) (Refs. 7-10) techniques supply nanosecond and subnanosecond dynamical information along with spatial images of moving magnetization.

In mesoscopic and nanoscale flat patterned elements (dots) made from soft magnetic materials, strongly nonuniform "vortex" magnetization configurations are typically observed at equilibrium. A magnetic vortex consists of an inplane flux-closure magnetization distribution with a very small central core that is magnetized perpendicularly to the dot plane. ${ }^{11-13}$ The vortex state can be characterized by a core polarization $p= \pm 1$ that describes whether the core is oriented up or down. It was demonstrated by spin-polarized scanning tunneling microscopy that the core radius is $\sim 10 \mathrm{~nm}$, i.e., about the material exchange length. ${ }^{14}$ Because the moving magnetic vortex core (VC) experiences a topological force (gyroforce) perpendicular to its velocity, ${ }^{15,16}$ the magnetic vortices in restricted geometry exhibit unique dynamic excitations. One of them corresponds to gyrotropic spiral-like motion of the vortex core around an equilibrium position with an eigenfrequency of the order of several hundred megahertz. ${ }^{4.17}$ The vortex core polarization via gyrovector determines the direction of the core spiral motion, as demonstrated in time-resolved magneto-optical $\mathrm{Kerr}^{4,6}$ and XMCD experiments. ${ }^{7.9 .10}$ The moving vortex profile is distorted in comparison to the static vortex ${ }^{1.18}$ due to interaction of the gyrotropic mode with azimuthal spin waves., ${ }^{19,20}$ This distortion increases when the core velocity increases, eventually leading to core polarization reversal. ${ }^{10.21}$ It has been shown that the vortex core polarization can be reversed by the application of a small ac magnetic field with a vortex resonance frequency and an amplitude considerably smaller than the switching field of the vortex core static reversal. ${ }^{22}$ This effect was experimentally observed ${ }^{10}$ and confirmed by micromagnetic simulations..$^{23.24}$ A universal criterion for vortex core switching has been formulated in terms of the vortex core critical velocity. ${ }^{25}$

Magnetic systems are essentially nonlinear due to a specific form of the Landau-Lisfitz-Gilbert equation of motion governing their dynamics. The magnetization dynamics exhibit phenomena, such as bifurcations and chaos, proper of nonlinear dynamical systems. ${ }^{26.27}$ The nonlinear effects manifest themselves when the excitation strength (external field or spin-polarized current value) is increased. In magnetism they were first observed in high-power ferromagnetic resonance experiments in the 1950 s (Refs. 28-30) and were explained by Suhl. ${ }^{31}$ These initial studies of nonlinear dynamics were focused on a saturated (almost uniform) magnetization state. More recently, nonuniform magnetic systems have been shown to exhibit a wealth of other interesting 
phenomena, such as spin-wave self-focusing, ${ }^{32}$ spin-wave localization, ${ }^{33}$ symmetry-breaking dynamic Bose-Einstein condensation of spin waves, ${ }^{34}$ magnetization reversal, driven by spin-wave instabilities, ${ }^{35.36}$ fold-over and bistability effects. ${ }^{26,37}$ An attempt to study the vortex nonlinear dynamics has been presented in Ref. 38, where the splitting of microwave absorption peaks corresponding to the vortex gyrotropic mode has been observed increasing the external field amplitude. This was explained by the occurrence of a foldover bifurcation, i.e., the appearance of an additional stable vortex orbit.

In this paper, we present calculations of the lowfrequency (subgigahertz range) nonlinear vortex dynamic excitations in submicron-size circular soft magnetic dots. For simplicity, we use a circularly polarized driving magnetic field so that the flipping between the states $p=+1$ and $p=$ -1 is suppressed. We briefly discuss the difference with a linearly polarized field, where multiple vortex core switching is possible. The observed low-frequency oscillations of the VC position are described as a gyrotropic motion of the VC around its equilibrium induced by a gyroforce and dynamic magnetostatic restoring forces in Sec. II. We derive analytical equations of the VC field-driven motion in the nonlinear regime in Sec. III. We describe nonlinear vortex dynamics in two regimes: the stationary regime (Sec. IV), showing the fold-over bifurcation and the nanosecond (transient) regime (Sec. V), showing the transient oscillations of the VC radius. Depending on the parameter values, the occurrence of the fold-over bifurcation and the nonlinear resonance may be suppressed by the vortex core reversal described in Sec. VI. Our results summarized in Sec. VII unambiguously demonstrate the importance of nonlinear effects in the magnetic vortex dynamics with a rich behavior still awaiting clear experimental demonstration.

\section{MODEL}

The approach is based on the Landau-Lifshitz-Gilbert equation of motion for the classical magnetization field $\mathbf{M}(\mathbf{r}, t)$,

$$
\dot{\mathbf{m}}=\gamma \mathbf{m} \times \delta w / \delta \mathbf{m}+\alpha \mathbf{m} \times \dot{\mathbf{m}},
$$

where $\mathbf{m}=\mathbf{M} / M_{s}$ is the reduced magnetization in units of the saturation magnetization $M_{s}, \gamma$ is the gyromagnetic ratio, and $\alpha$ is the Gilbert damping parameter. We use the magnetic energy density $w[\mathbf{m}(\mathbf{r}, t)]$ and the substitution $\mathbf{m}(\mathbf{r}, t)$ $=\mathbf{m}[\mathbf{r}, \mathbf{X}(t)]$, where $\mathbf{X}=(X, Y)$ defines the VC position. Upon integration over the current coordinates $\mathbf{r}$, the energy density $w[\mathbf{m}(\mathbf{r}, t)]$ becomes a function of the vortex core position $W(\mathbf{X})$. The motion of a vortex has traditionally been considered analytically in the limit of small excitations using the Thiele's equation of motion ${ }^{15,17}$ for $\mathbf{X}$, in combination with an energy function $W(\mathbf{X})$ for a given dot geometry. Including a damping force, ${ }^{30,40}$ this equation can be written as

$$
-\mathbf{G} \times \dot{\mathbf{X}}-\hat{D} \dot{\mathbf{X}}+\frac{\partial W(\mathbf{X})}{\partial \mathbf{X}}=0
$$

where a dot over a symbol denotes a derivative with respect to time and $\hat{D}$ is a damping tensor, ${ }^{15,39}$ which is diagonal for a circular dot. The first term, the gyroforce, is proportional to the gyrovector $\mathbf{G}=-G q p \hat{\mathbf{z}}$, where $G=2 \pi L M_{s} / \gamma, L$ is the dot thickness and $\hat{\mathbf{z}}$ is the unit vector perpendicular to the dot plane. In the linear limit assuming the decomposition $W(\mathbf{X})$ $=W(0)+(\kappa / 2)|\mathbf{X}|^{2}, \mathbf{E q}$. (2) can be easily solved analytically, yielding the vortex eigenfrequency $\omega_{0}=G^{-1} \kappa$, calculated by Guslienko et al. ${ }^{17}$

Topological charges such as vorticity $(q)$ and polarization (p) determine the vortex gyrovector, essential for the vortex dynamics. ${ }^{18}$ The dimensionless damping is $d=-D /|G|>0$. We assume that the damping coefficient $d \ll 1$ is small and neglect the nonlinearity of the damping term. Considering only the motion of the vortex core coordinate $\mathbf{X}$, we neglect also the vortex-spin waves interaction, which would lead to the appearance of a finite but relatively small vortex mass. ${ }^{20}$

The pole-free model ${ }^{17}$ of moving vortex uses a representation of magnetization $\mathbf{m}(x, y)$ via a complex function $w(z, \bar{z})=\left(m_{x}+\mathrm{i} m_{y}\right) /\left(1+m_{z}\right), w(z, \bar{z})=f(z)$ if $|f(z)| \leq 1$ and $w(z, \bar{z})=f(z) / f(z) \mid$ if $|f(z)|>1 .^{18}$ The analytical function $f(z)=\mathrm{i}\left(C / R_{c}\right)\left[z-\left(Z+\bar{Z} z^{2}\right) /\left(1+|Z|^{2}\right)\right]$ describes the shifted vortex magnetization distribution in the dot as superposition a vortex centered in the point $z_{1}=Z$ and "image" vortex centered in the point $z_{2}=1 / \bar{Z}$. Here $z=x+i y, x, y$ are in units of $R, Z=(X+\mathrm{i} Y) / R$ describes the vortex core position $\mathrm{X}$, and $R_{c}$ is the vortex core radius.

The vortex restoring force $-\partial W(\mathbf{X}) / \partial \mathbf{X}$, responsible for the finite gyrotropic frequency, is mainly defined by the magnetostatic energy term, with the exchange energy providing a smaller contribution. ${ }^{17.18}$ In the case of field-driven dynamics, the Zeeman energy must also be included. In Refs. 17 and 38 , the dipolar energy contribution was shown to be quadratic for small perturbations but a fourth-order term is required for larger core displacements, leading to the quartic form

$$
W_{\text {dem }}(\mathbf{X})=(\kappa / 2)|\mathbf{X}|^{2}+(\beta / 4)|\mathbf{X}|^{4}
$$

for a circular dot (an elliptical dot would require an anisotropy in $\mathrm{X}$ and $\mathrm{Y}$ components and corresponding stiffness coefficients). The Zeeman energy is also nonlinear

$$
W_{Z}(\mathbf{X})=-\int d V \mathbf{M} \cdot \mathbf{H}=-C(\hat{\mathbf{z}} \times \mathbf{h}) \cdot \mathbf{X}\left[\xi-\delta|\mathbf{X}|^{2}\right],
$$

where $C$ is the vortex chirality, $\xi$ and $\delta$ are constants which can be calculated from the volume average of the in-plane magnetization components $m_{x}, m_{y}$ and $\mathbf{h}=\mathbf{H} M_{s} V$. We assume that the in-plane driving ac field of frequency $\omega$ is circularly polarized and the parameter $h(t)$ represents the field in complex number notation if $H(t)=H_{0} e^{i \text { int }}$, then $h(t)=H(t) M_{s} V$.

The nonlinear coefficients can also be calculated within the pole-free model, ${ }^{9.17}$ and their signs are $\beta>0, \delta>0$. The linear response coefficient is $\xi=2 /(3 R)$ ( $R$ is the dot radius) within the same model. ${ }^{17}$ It can be shown that the nonlinearity of the Zeeman energy $(\delta)$ is small in comparison with the nonlinearity of the magnetostatic energy $(\beta)$. This corresponds to approximation $H_{0} \ll H_{\text {an }}$ ( $H_{\text {an }}$ is the vortex annihilation field) and only the nonlinearity of the system due to nonzero $\beta$ will be accounted for in the next sections. The positive sign of $\beta$ was confirmed by micromagnetic 
simulations. ${ }^{41,42}$ According to Ref. 42, the nonlinearity of $W_{\text {dem }}(\mathbf{X})$ is essential at $|\mathbf{X}|>0.13 R$. We will use the field amplitude $h_{0}$ in units of the linear eigenfrequency $\omega_{0}$, $h_{0} / \omega_{0}=\gamma H_{0} / 3 \omega_{0}$. In what follows, where not explicitly indicated, we use the following typical values of the parameters: the damping $d=0.03, \omega_{0} /(2 \pi)=(100-500) \mathrm{MHz}$, and $\beta$ $=4 \omega_{0}$. For simplicity we consider in the next sections the vortex core up case with $p=1$.

\section{DYNAMICAL SYSTEM FOR VORTEX-CORE TRAJECTORIES}

Let us consider analytically the problem of nonlinear vortex motion in a circular dot. It is convenient to write the nonlinear equations of motion $[\mathrm{Eq} .(2)]$ in terms of the amplitude $u$ and phase $\Phi$ of the complex variable $Z(t)$ representing the vortex core position. In a coordinate frame rotating with the field frequency $\omega$,

$$
Z(t)=u(t) e^{i[\phi(t)+u n t]} .
$$

Note that the vortex orbit radius $|Z(t)|=u(t)$ and the phase difference $\phi(t)$ between the external field $h(t)=h_{i} e^{i(\theta t}$ and the vortex response depend, in general, on $t$.

The contesponding dynamical system takes the form

$$
\begin{gathered}
\dot{\phi}=-\omega+\frac{1}{1+d^{2}}\left[h_{0}(d \cos \phi-\sin \phi) \frac{1}{u}+\beta u^{2}+\omega_{0}\right], \\
\dot{u}=\frac{1}{1+d^{2}}\left[h_{0}(\cos \phi+d \sin \phi)-d\left(\beta u^{2}+\omega_{0}\right)_{u}\right] .
\end{gathered}
$$

We get in the linear limit $(\beta=0)$ the solution as a sum of "free" motion and field-driven parts,

$$
Z(t)=\left[Z(0)-\frac{i h_{0}}{\Omega_{0}-\omega}\right] e^{i \omega t_{0} t} e^{-d\left(t_{0} t\right.}+\frac{i h_{0}}{\Omega_{0}-\omega} e^{i(t) t},
$$

where $\Omega_{0}=\omega_{0}+i d \omega, Z(0)=Z(t=0)$ is the initial vortex core position. System (6) has an integral of motion,

$$
h_{0}^{2}=[\dot{u}+d(\dot{\phi}+\omega) u]^{2}+\left[\left(-\dot{\phi}-\omega+\omega_{0}+\beta u^{2}\right) u+d \dot{u}\right]^{2} .
$$

Stationary solutions satisfy $\dot{\phi}=\dot{i}=0$, and correspond to circular vortex core motions with fixed orbit amplitude $u=u_{0}$, usually detectable experimentally, and fixed phase difference $\phi=\phi_{0}$. Then we say that the dynamical system (6) has a stationary or critical point $\left(u_{0}, \phi_{0}\right)$ in phase space. For them Eq. (7) becomes

$$
\left(\beta u^{2}-\omega+\omega\right)_{0}^{2} u^{2}+d^{2} \omega^{2} u^{2}=h_{0}^{2},
$$

a polynomial equation on $u^{2}$. Its roots give the stationary amplitudes $u_{i}$, and $\phi_{0}$ can be retrieved from $\mathrm{Eq}$. (6).

The stability of a stationary solution $\left(u_{0}, \phi_{0}\right)$ is determined by linearizing the dynamical system (6) around it, i.e., taking $u=u_{0}+\delta u, \phi=\phi_{0}+\delta \phi$ obtaining

$$
\left(\begin{array}{c}
\delta \dot{\phi} \\
\delta \dot{u}
\end{array}\right)=M\left(\begin{array}{c}
\delta \phi \\
\delta u
\end{array}\right)
$$

where
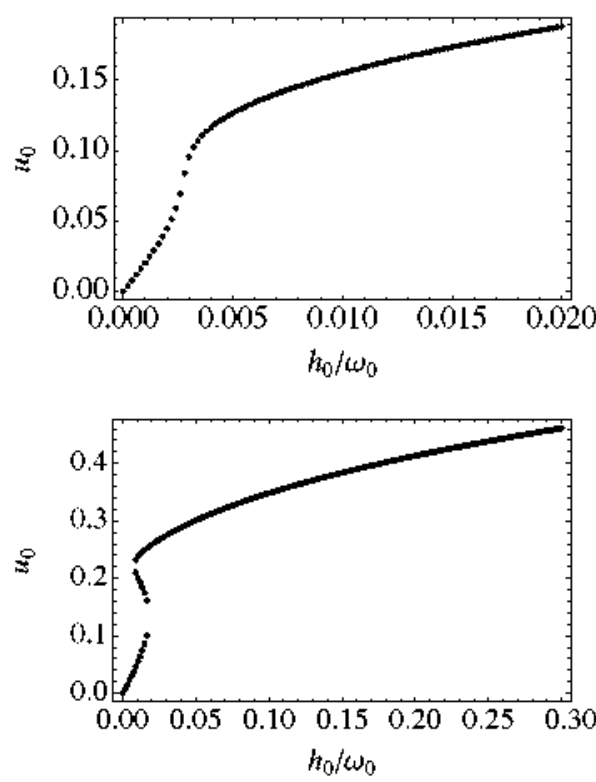

FIG. 1. (Color online) Amplitude of the vortex core stationary orbits vs amplitude of the external field $\left[\omega_{0} /(2 \pi)=250 \mathrm{MHz}\right]$

$$
M=\left[\begin{array}{cc}
-d\left(\beta u_{0}^{2}+\omega_{0}\right) & \frac{3 \beta u_{0}^{2}-\left(d^{2}+1\right) \omega+\omega_{0}}{u_{0}} \\
u_{0}\left(\omega d^{2}-\beta u_{0}^{2}+\omega-\omega_{0}\right) & -d\left(3 \beta u_{0}^{2}+\omega_{0}\right)
\end{array}\right] .
$$

Standard dynamical system theory establishes that the character of the stationary solution is given by the invariants of the matrix $M$,

$$
\begin{gathered}
\operatorname{tr} M=-2 d\left(2 \beta u_{0}^{2}+\omega_{0}\right), \\
\operatorname{det} M=\left(d^{2}+1\right)\left[\left(2 \beta u_{0}^{2}-\omega+\omega_{0}\right)^{2}+d^{2} \omega^{2}-\beta^{2} u_{0}^{4}\right], \\
\operatorname{discr} M=4\left[\left(1+d^{2}\right) \beta^{2} u_{0}^{4}-\left(2 \beta u_{0}^{2}-\omega d^{2}-\omega+\omega_{0}\right)^{2}\right],
\end{gathered}
$$

where $\operatorname{discr} M=(\operatorname{tr} M)^{2}-4$ det $M$ and the eigenvalues are

$$
\lambda^{ \pm}=\frac{\operatorname{tr} M}{2} \pm \frac{\sqrt{\operatorname{discr} M}}{2} .
$$

Equation (8) has only one root for every value of $h_{0}$ if $\left|\omega-\omega_{0}\right|<\sqrt{3} d \omega$. If $\omega-\omega_{0}>\sqrt{3} d \omega$ it can have three roots but only for the following range of values of $h_{0}$,

$$
\begin{aligned}
\frac{2}{27 \beta}\left[\nu^{3}+9 d^{2} \omega^{2} \nu-2 \sqrt{\left[\nu^{2}-3 d^{3} \omega^{2}\right]^{3}}\right]<h_{i}^{2} \\
<\frac{2}{27 \beta}\left[\nu^{3}+9 d^{2} \omega^{2} \nu+2 \sqrt{\left[\nu^{2}-3 d^{3} \omega^{2}\right]^{3}}\right] .
\end{aligned}
$$

Figure 1 depicts, for two fixed values of $\omega>\omega_{0}$, the values $u_{0}$ of $u$ satisfying Eq. (8), depending on $h_{0}$. With $\omega$ $=1.04 \omega_{0}$ condition (10) does not hold and there is only one, stable, stationary circular orbit of the vortex core for any $h_{0}$. With $\omega=1.2 \omega_{0}, \mathrm{Eq}$. (10) holds for some values of $h_{i,}$ for which there are three stationary VC orbits, two stable and one unstable; outside this range of $h_{i}$ there is again only one, stable, stationary VC orbit. The folding of the curve in Fig. $1(\mathrm{~b})$ is known as fold-over bifurcation. The same effect hap- 

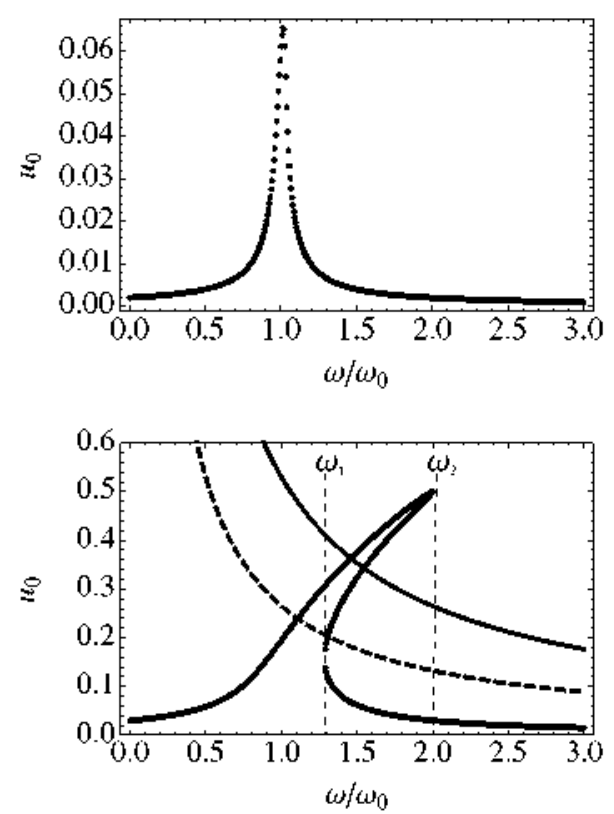

FIG. 2. (Color online) Amplitudes of the stationary orbits vs the extemal field frequency for $\omega_{0} /(2 \pi)=250 \mathrm{MHz}$. The hyperbolas indicate the critical values of the vortex velocity above which core reversal occurs for $L_{e}=L$ (solid line) and $L_{e}=L / 2$ (dashed line), see Sec. V.

pens when we only change, instead of the external field amplitude $h_{0}$, the external frequency $\omega$, see Fig. 2 .

In Fig. 3 we present in the $\phi-u$ plane the phase portraits of the dynamical system (6) in two cases: one or three stationary solutions. The accessibility of one or another stable stationary VC orbit depends on the initial conditions. Note the following hysteresis process related to the transition between the two orbits, illustrated in Fig. 2(b). In the broadband ferromagnetic resonance experiments on vortex dynamics, ${ }^{1.3 .6}$ the external frequency $\omega$ is swept through a given range. Starting with the vortex in the dot center, the vortex orbit increases in amplitude with $(t)$, becoming unstable at frequency $(\omega)=(\omega)$, where it abruptly decreases its amplitude. After that the amplitude further decreases with $\omega$. If then the frequency is decreased back, the amplitude of the vortex orbit slowly increases. At $\omega=\omega_{1}<\omega_{2}$, the orbit becomes unstable again and the vortex abruptly passes to a new orbit with larger amplitude. A similar hysteresis happens when sweeping in the external field amplitude $h_{0}$.

\section{QUASISTATIONARY REGIME AND RESONANCE CONDITIONS}

We consider in this section the quasistationary regime, reached after some hundreds of nanosecond in the experiments. The system settles in one of the stable stationary points $\left(u_{i j}, \phi_{i j}\right)=\left(u_{i}, \phi_{i}\right), i=1,2$, corresponding to the roots of Eq. (8) and the motion of the vortex core is a circular oscillation $Z(t)=u_{i} e^{i u t+\phi_{i}}$. The absorbed power in resonance experiments is proportional to the squared vortex core amplitude. This power will be maximum when the amplitude of the trajectory is maximum (see Fig. 2).
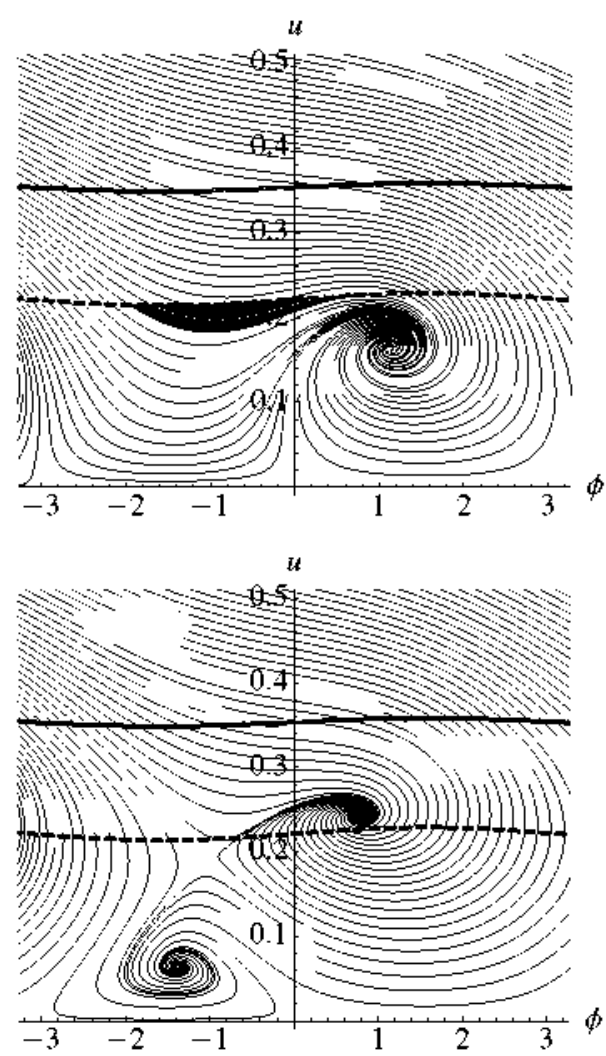

FIG. 3. (Color online) Phase space of Eq. (6) when $\omega_{0} /(2 \pi)$ $=250 \mathrm{MHz}$ and $h_{0}=0.012 \omega_{0}$. The lines indicate the critical values of the vortex velocity above which the core reversal occurs for $L_{e} / L=1$ (solid line) and $L_{e} / L=0.5$ (dashed line), see Sec. V. The shaded region is composed of trajectories that are beyond the reversal curve but also go to vortex core reversal.

The frequency $\left(_{2}\right.$ of the maximum of the first root can be calculated with a differentiation argument considering $u_{i}$ as a function of $(\omega$, and differentiating $\mathrm{Eq}$. (8) with respect to $(t)$ Then the maximum of $u$ is reached when $u^{\prime}(\omega)=0$. The result is

$$
\left(\omega_{2}-\omega_{i}\right) \omega_{2}^{2}+d^{2} \omega_{2}^{3}=\frac{h_{i}^{2} \beta}{\left(1+d^{2}\right) d^{2}} .
$$

The frequency $\omega_{1}$ of the maximum of the second root can be calculated solving the bifurcation condition,

$$
\begin{gathered}
27 \beta^{2} h_{0}^{4}-4 \beta\left(\omega_{1}-\omega_{0}\right)\left[\left(9 d^{2}+1\right) \omega_{1}^{2}-2 \omega_{0} \omega_{1}+\omega_{0}^{2}\right] h_{\phi}^{2} \\
+4 d^{2} \omega_{1}^{2}\left[\left(d^{2}+1\right) \omega_{1}^{2}-2 \omega_{0} \omega_{1}+\omega_{0}^{2}\right]^{2}=0 .
\end{gathered}
$$

This equation can be easily solved numerically and analytically at $d=0$ yielding the frequency $\omega_{1}=\omega_{0}+3\left(2 \beta h_{i}^{2}\right)^{1 / 3} / 2$. We present a plot of the resonant $\omega$ 's versus $h_{0}$ in Fig. 4 (solid lines).

As it can be seen, the main consequence of the nonlinear vortex dynamics is the appearance of the splitting of the main resonant line into two resonant peaks, corresponding to the fold-over bifurcation. However, one should note that the experimental detection of the second resonance line, sweeping the frequency, may be difficult due to the small amplitude $\left(u_{1} \sim 0.1\right)$ of the smaller vortex orbit. 


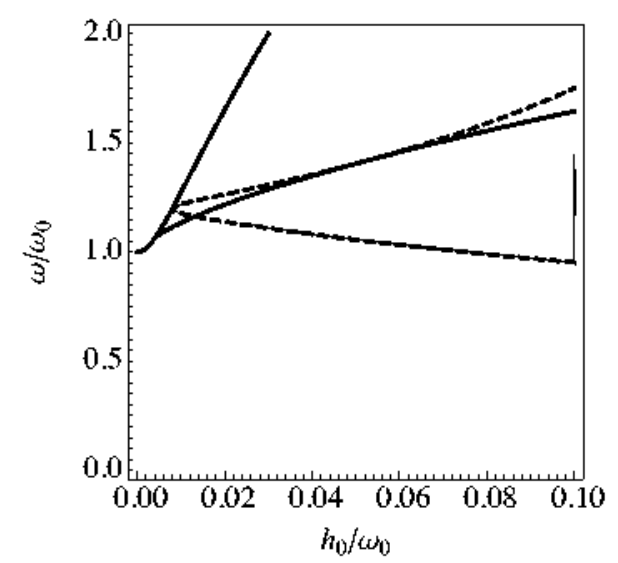

FIG. 4. (Color online) Resonance diagram for $\omega_{0} /(2 \pi)$ $=250 \mathrm{MHz}$. The solid line depicts the resonance conditions while the dashed line shows when the critical orbits reach VC reversal (estimated for $L_{e}=L / 2$ ). The area between the dashed lines indicates the region of the vortex core reversal, see Sec. V.

The second resonance appears at $h_{0}>h_{i,}^{\text {cr }}$, where the critical values are

$$
\omega^{\mathrm{cr}}=\frac{\omega_{0}}{1-\sqrt{3} d}, \quad h_{0}^{\mathrm{cr}}=\frac{2 \sqrt{2}}{\sqrt[4]{27}}\left[\frac{d \omega_{0}}{1-\sqrt{3} d}\right]^{3 / 2} \frac{1}{\sqrt{\beta}} .
$$

The finite value of $\beta$ results in a finite value of the threshold field $h_{0}^{\mathrm{cr}}$, which is typically about of $1 \mathrm{Oe}$. Thus it should be possible to determine experimentally the vortex damping $d$ and nonlinearity $\beta$ by measuring these two critical values. But we need also to account for the vortex core reversal which occurs increasing $h_{0}$ for a given $\omega$, see details in Sec. VI. The minimal field for reversal is $h_{r}=3 d v_{c} / \gamma R \sim 10 \mathrm{Oe} .^{25}$ Both frequencies $\omega_{1}, \omega_{2}$ exist within the field range $h_{i}^{c r}<h_{0}$ $<h_{r}(\omega)$, where the dependence $h_{r}(\omega)$ is plotted by the dashed line in Fig. 4 for some particular value of the dot thickness. The frequency $\omega_{2}$ has no sense at $h_{0}>h_{r}\left(\omega_{2}\right)$ (the shadowed area in Fig. 4) because the vortex core is reversed and the circular driving field is not effective anymore to excite the vortex motion.

\section{NANOSECOND TIME SCALE (TRANSIENT) NONLINEAR VORTEX DYNAMICS}

Before the onset of the stationary regime after the ac field is switched on, there is an interval between tens and hundreds of nanoseconds (a time $t_{r}$ of about of $1 / d \omega_{0}$ ) where a transient regime of vortex motion takes place. The position of the vortex core obeys the dynamical system (6), and starting from any initial position, it spirals down into one of the stable stationary orbits described in previous sections, i.e., with the amplitude $u_{i}$ and phase $\phi_{i}$ that depend on the parameters $h_{0}$ and $\omega$, see Fig. 3. This kind of dynamics is nowadays accessible by several experimental techniques, such as XMCD measurements ${ }^{7,810}$ and time-resolved Kerr effect ${ }^{4-6}$ Due to the limitations of the total integration time, this are also the dynamics studied in numerical experiments, by integrating the Landau-Lifshitz-Gilbert ${ }^{2+25,41,43}$ or the Thiele's equations of motion. ${ }^{41}$

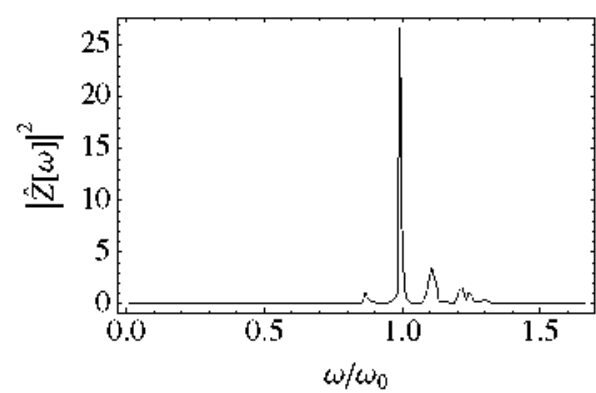

FIG. 5. (Color online) Transient spectrum of the vortex motion with $\omega_{0} /(2 \pi)=100 \mathrm{MHz}, \omega=1.2 \omega_{0}, h_{0}=0.012 \omega_{0}$, and $d=0.003$.

To illustrate the transient vortex dynamics, we display in Fig. 5 the square of the amplitude of the Fourier transform $\hat{Z}(\omega)$ of the transient motion of the vortex $Z(t)$ near an stationary focus with larger orbit radius $u=u_{2}$. Besides the principal peak at $\omega$, additional peaks appear.

The analytical description for the nonlinear vortex dynamics in this regime is possible in two limiting cases. In the first case, we neglect vortex damping. Then, we get from Eq. (6) with $d=0$ the following nonlinear system of autonomous equations of motion:

$$
\begin{gathered}
\left(-\dot{\phi}-\nu+\beta u^{2}\right) u=h_{0} \sin \phi, \\
\dot{u}=h_{0} \cos \phi
\end{gathered}
$$

where $\nu=\omega-\omega_{i j}$. These equations can be integrated explicitly introducing the total dynamic vortex energy integral of motion [Eq. (7)]. It can be shown that the solution of the system (11) can be written in closed form as

$$
\eta(t)=\eta_{1}+\frac{1}{4}\left[\frac{g^{\prime}\left(\eta_{1}\right)}{\rho\left(2 t \mid g_{2}, g_{3}\right)-\frac{1}{24} g^{\prime \prime}\left(\eta_{1}\right)}\right],
$$

where $\eta(t)=u^{2}(t), \eta_{1}=\eta(0), g(\eta)=h_{0}^{2} \eta-\left(H+\nu \eta / 2-\beta \eta^{2} / 4\right)$, and $\rho\left(z \mid g_{2}, g_{3}\right)$ is the Weierstrass special function (see Ref. 44). The vortex orbit $u(t)$ is a periodic function of time with period $T$ and eigenfrequency $\omega_{t}=2 \pi / T$. The period $T$ and the Weierstrass invariants $g_{2}, g_{3}$ can be explicitly calculated as functions of the parameters $h_{0}, \nu, \beta$, and $H$. The phase $\Phi(t)$ is also a periodic function of time with period $T$. Then, the vortex core positions $X$ and $Y$ as functions of time oscillate with two frequencies $\left(\omega, \omega_{t t}\right)$ and are determined by equations,

$$
\begin{gathered}
X(t) / R=u(t) \cos [\omega t+\phi(t)], \\
Y(t) / R=u(t) \sin [\omega t+\phi(t)],
\end{gathered}
$$

i.e., the vortex core oscillates within the ring $\sqrt{\eta_{1}} \leq|u(t)|$ $\leq \sqrt{\eta_{2}}$ with the eigenfrequency $\omega_{t u}=2 \pi / T$, which is different from the field driving frequency o and the vortex linear eigenfrequency $\omega_{0}$. The value of $\omega_{t i}$ may be smaller than $\omega_{0}$ (for $\nu=0$ ), or bigger than $\omega_{0}$ depending on the parameters $h_{0}$, $\nu$, and $\beta$. Consequently, the vortex motion is basically a superposition of two motions with frequencies $\omega_{u}$ and $\omega$, and 
the vortex trajectory may be very complicated in the nonlinear regime $(\beta \neq 0)$.

The second case is, retaining the magnetization damping, to consider a motion close to the stationary orbits. The dynamics at the nanosecond scale depend on the character of the stationary point that the system will ultimately reach given its initial state. This character is determined by the linearized system around the critical point or, more concretely, by the eigenvalues [Eq. (9)] of the matrix $M$. The trace is always negative, and the decisive quantity is the sign of the discriminant. If discr $M \geq 0$ we have a stable node, and the vortex core goes exponentially fast to the stable circular motion. The situation is more interesting if discr $M$ $<0$ : we have a stable focus, and there is an additional oscillation of certain frequency $\omega_{3}$ with amplitude exponentially decaying around the final stationary motion of the core. The eigenvalues are $-\lambda \pm i \omega_{3}$ with $\lambda=-\operatorname{tr} M / 2$ and $\omega_{3}$ $=\sqrt{-\operatorname{discr} M} / 2$ and the moving vortex core trajectory is

$$
Z(t)=\left(u_{i}+u_{j} e^{-\lambda t} \cos \left(\omega_{3} t\right) e^{i\left(s_{0} t+\phi_{i}+\left(t_{p} t_{3} ; / t_{0}\right) e^{-\lambda t} \sin u_{i} t\right)},\right.
$$

where $u_{i}$ is the small core oscillation amplitude (cf. Fig. 3 where all the stable points are focuses). Let us suppose, for simplicity, that we always start the trajectory at a point where $\phi(0)=\phi_{0}$ and $u(0)=0$. Using the Fourier series $\left[J_{n}(z)\right.$ are Bessel functions],

$$
e^{i i t \theta+z \sin \phi !}=\sum_{n=-\infty}^{n=\infty} J_{n}(z) e^{i(\theta+n \phi)}
$$

and developing $Z(t)$ in series, we derive the existence of additional frequency peaks at $\omega \pm \omega_{3}, \omega \pm 2 \omega_{3}$, etc. Thus, the transient oscillation adds additional components or frequency "peaks" to the measured spectrum of the vortex, in the nanosecond scale. In fact, due to frequency locking between the radius and the phase oscillations, there are two prominent peaks in the spectrum, one at $\omega$ and another one at $(t)+\omega_{3}$. This effect is more accentuated in the case $d=0$. The frequency $\omega_{3}$ at $d=0$ is simply the small amplitude approximation of the nonlinear frequency $\omega_{u}, \omega_{3}(d=0)=\omega_{u}$ near the stable points. Note that in the laboratory frame, the stable focus $(\dot{u}=0, \dot{\phi}=0)$ is a limiting cycle of the vortex core trajectory $|Z(t)|=$ const and therefore, its radius is oscillating in time. The amplitude of the additional harmonics decay exponentially in time with the relaxation time $t_{r} \sim 1 / d \omega_{0}$.

\section{NONLINEAR EFFECTS AND VORTEX-CORE REVERSAL}

The approach given above uses the pole-free model of the dynamic vortex and consequently is valid only before large distortions in vortex shape occur. According to recent studies, ${ }^{45}$ these distortions occur for vortex core shifts of $X$ (or $Y$ ) $\sim 0.2 R$. Definitely, our approach loses its validity when reversal of the vortex core occurs and the gyrovector $G$, component changes its sign. According to the universal criterion, ${ }^{25}$ this occurs when the vortex core reaches a critical velocity $v_{c} \sim 1.66 \gamma \sqrt{A}$, where $A$ is the exchange stiffness or in terms of our notations (in units of $\omega_{i j} R$ ),

$$
v_{c}=0.530\left(L_{e} / L\right) \text {, }
$$

and $L_{e}=\sqrt{2 A / M_{s}^{2}}$ is the exchange length (18 nm for permalloy), $L$ is the dot thickness, and the expression $\omega_{0}$ $=(20 / 9) \gamma M_{s} L / R$ was used. ${ }^{9}$ The existence of such critical velocity $\left(v_{c}=250 \mathrm{~m} / \mathrm{s}\right.$ for permalloy dots) was recently confirmed by time-resolved magnetic scanning transmission $\mathrm{x}$-ray microscopy ${ }^{46}$ for vortex core reversal in FeNi dots. We used in Figs. 2 and 3 the values $L_{e} / L=1 / 2$ and 1 to consider typical dot thicknesses. We assume that the dot radius is big enough $(R>100 \mathrm{~nm})$ to avoid the mechanism of vortex core reversal via its escape from the dot and return with opposite core polarization. ${ }^{47}$

The vortex velocity $v=|\dot{Z}(t)|$ is well defined in every point of the phase space $(\phi, u)$ because

$$
|\dot{Z}(t)|^{2}=\dot{u}(t)^{2}+[\omega+\dot{\phi}(t)]^{2} u(t)^{2}
$$

or, using Eq. (6), $|\dot{Z}(t)|^{2}=\frac{1}{1+d^{2}}\left\{\left[h_{0}-\left(\beta u^{2}+\omega_{0}\right) u\right]^{2}+2 h_{0}\left(\beta u^{2}\right.\right.$ $\left.\left.+\omega_{i}\right) w(1-\sin \phi)\right\}$. The phase space is then divided into two regions: (1) the region where $v<v_{c}$, let us call it allowed region; (2) the region where $v>v_{c}$, where the core reversal occurs and our model ceases to be applicable.

We call the curve $v=v_{c}$ separating both regions the reversal curve. We suppose that the initial state of the vortex $(t=0)$ is in the allowed region. In terms of the dynamical system, we have to search for phase trajectories that touch the reversal curve, i.e., vortices that reach the reversal velocity under influence of the driving ac field. In fact, it is possible to determine this region numerically, as depicted in Fig. 3(a).

In Fig. 3, we present an illustration of several possible situations. It can happen that no vortex that starts in the allowed region goes to reversal. In this case, all the trajectories that start in the reversal curve continue into the allowed region, without returning to the reversal curve. For some critical values of the external field and frequency, reversal trajectories appear. For example, in Fig. 3, when the reversal curve is the solid one $\left(L_{e} / L=1\right)$ all trajectories in the allowed region lead to the stable stationary point when $t$ increases. If the reversal curve is the dashed one $\left(L_{e} / L=1 / 2\right)$ then, if $\omega$ $=1.04 \omega_{0}$ [Fig. 3(a)], albeit the stable stationary point is in the allowed region, there are some trajectories just under the reversal curve, around $\phi=-1$, that would lead to vortex reversal. If $\omega=1.2 \omega_{0}$ [Fig. 3(b)] the system has two stable points, the lower being in the allowed region and the higher in the reversal region. Basically, trajectories belonging to the basin of attraction of the higher stable point (those trajectories that end up in the higher stable point) will produce the vortex core reversal. Conversely trajectories in the basin of attraction of the lower stable point will not produce reversal.

The regions of the parameters where the core reversal takes place can be derived analytically in the quasistationary regime. We assume that the external parameters change very slowly with respect to the fast dynamics that make the vortex core go to the stable point. Consequently the VC always stays at the stationary orbit, which slowly moves on the phase space with the change in parameters. At the beginning of the process, we suppose that the stable point is in the 
allowed region $v<v_{c}$, and then some parameter is increased, making the VC orbit (stable point) approach the reversal curve. Reversal takes place when the stable point reaches the reversal curve. Evidently [cf. Eq. (14)] the squared velocity of the VC at the stationary point is $|\dot{Z}(t)|^{2}=\omega^{2} u_{i}^{2}$, therefore, the condition of reversal in the quasistationary regime is

$$
v_{c}^{2}=\omega^{2} u_{i}^{2}
$$

i.e., in this case the condition for vortex core reversal can be reformulated in terms of the maximum radius of the core trajectory, as in Ref. 25. Condition (15) can be easily plotted in a $\omega$ - $u$ diagram, as in Fig. 2(b), where we have drawn two curves, the solid one corresponding to $L_{e} / L=1$ and the dashed one to $L_{e} / L=1 / 2$. One can see that in an adiabatic process, the vortex follows a stable orbit while (t) is small. When $\omega$ reaches a critical value $\omega_{r}=v_{c} / u_{i}$ such that the velocity of the vortex is the reversal velocity, reversal takes place. Reversal is accompanied by strong spin-wave emission and the system experiences resonance phenomena of a different nature than that discussed in Sec. IV. Since our field is circularly polarized, reversal of the vortex core stops the dynamics. In the resonance diagram of Fig. 4, we have depicted with a dashed line the condition of vortex core reversal in quasistationary regime, i.e., Eq. (15). In the shaded region, the vortex core polarization is reversed and the dynamics is suppressed under a circularly polarized field.

If the in-plane magnetic field is linearly polarized, a vortex with the opposite core polarization would couple to the field with opposite rotation sense and reveal similar dynamics. In this case after entering the core reversal region, increasing $\omega$ up to $\omega_{1}$ may lead to the creation of another possible stable orbit for the vortex with opposite polarization in the allowed region. However, it is not clear when the system acquires a stable vortex state again because, close to $\omega=\omega_{1}$, many possible trajectories of the vortex go to reversal. This corresponds to multiple reversals of the vortex core polarity in this region for most of the initial conditions. At the same time, some other trajectories go to the stable orbit that appeared in the nonreversing region. Increasing further $\omega$ would lead to a stable circular vortex motion in the nonreversing region, with a small radius.

\section{DISCUSSION AND CONCLUSIONS}

We have studied the vortex dynamics in circular ferromagnetic dots in terms of the general theory of nonlinear dynamical systems, showing the occurrence of stationary orbits undergoing a fold-over bifurcation. The stationary orbits appear as a result of the compensation of the damping by the energy supplied by the external ac field, a similar behavior as in spin-torque oscillators. In our study, we have distinguished between the quasistationary and nanosecond (transient) dynamics. The first type of dynamics is proper to experiments based on broadband ferromagnetic resonance, which senses the emitted power due to the vortex oscillation on stationary orbits. The second (transient) dynamics is proper to fast Kerr and XMCD measurements. Multiple frequency peaks (with intensity decaying in time) should be observed on this time scale. Numerical micromagnetics is also applicable to the transient region and additional frequency peaks and oscillating trajectory core radius have been reported. ${ }^{41}$ However, as was already noticed in this paper, most of the trajectories are transients and micromagnetic simulations experience serious difficulties in predicting long-time behavior.

We have calculated analytically the threshold values of the parameters for the fold-over bifurcation, showing how the splitting of resonant frequencies appears. This should also be manifested in hysteresis processes in experiments sweeping frequency or field. Originally in Ref. 38, the experimentally observed splitting of the resonant frequencies was attributed to the occurrence of this bifurcation. However, the observed splitting frequency is not in agreement with the value estimated by us, and both frequencies increase with external field, contrarily to the experimentally observed behavior. Second, the radius of the second orbit is rather small. Next, to demonstrate the occurrence of two stable solutions, in micromagnetic simulations one should start with initial conditions in the basin of attraction of different minima (frequently far from the dot center), which is not the experimental case. Finally, and most importantly, increasing the frequency, the largest orbit falls into the region of vortex core reversal. Consequently, we believe that the most plausible explanation of the resonant frequency splitting observed in Ref. 38 is the vortex core flipping. Indeed, strong emission of spin waves is known to occur during this process. The experimental validation of the fold-over bifurcation is still awaiting. Its definite proves should contain the observation of the hysteresis process while sweeping the field or the external frequency.

The consideration of nonlinear terms in the magnetic vortex dynamics reveals rich behavior, strongly dependent on initial conditions. The basin of attraction for the VC reversal, introduced above, shows the important role of the initial conditions, as is always the case in the theory of nonlinear dynamical systems. In micromagnetic simulations of the vortex core reversal, ${ }^{24.25,41,43}$ most frequently the initial condition with zero velocity (amplitude) and stationary phase $\left[\phi(t=0)=\phi_{0}, u(t=0)=0\right]$ are used. These conditions may produce core reversal while starting from the stationary solution $\left[u(t=0)=u_{i}\right]$ may not. The latter is more typical in the experimental situation.

We would like to mention here that experimentally the effect of the nonlinear vortex dynamics on its spectrum is possible to measure using the microwave reflection technique ${ }^{38}$ or the Hall effect ${ }^{48}$ and the direct observation of the vortex trajectories-by XMCD imaging. Whether it will be possible to directly measure the vortex phase aiming the reconstruction of the phase portraits of Fig. 3 is an open question. Additional common difficulties arise from the local defects and imperfect dot shape which may also influence the vortex dynamics via changing the local potential $W(\mathbf{X})$. We consider that influence of such defects can be essential for low-amplitude vortex dynamics (the trajectory radius $<10 \mathrm{~nm}$ ). But increasing the core orbit radius to the values of $50-100 \mathrm{~nm}$, much bigger than the defect size, the influence of the local defects is averaged and can be ruled out.

The importance of nonlinear effects in the vortex core dynamics goes beyond its concrete realization with circularly polarized ac field. For example, nonuniform magnetization 
(vortex) dynamics under the influence of a spin-polarized current is now being actively explored in different spintronic devices (namocontacts, nanopillars, and magnetic tumnel junctions). A question still under debate ${ }^{49}$ is the proper account of the spin-torque term in the Thiele equation of motion [Eq. (2)] for the vortex center $\mathbf{X}$. The spin-torqueinduced magnetization dynamics in nanopillars and tunnel junctions is essentially nonlinear and the approach developed here can be applied to plan and interpret such experiments. For instance, an alternative explanation of the magnetization dynamics induced by the spin-momentum transfer, in the $\mathrm{FeNi} / \mathrm{Cu} / \mathrm{FeNi}$ nanopillars ${ }^{50}$ is the existence of two stable solutions (two fold-over resonance frequencies) for the dynamics of a single vortex in the free layer, on a certain range of applied spin-polarized electrical current. The small fre- quency difference $(10-30 \mathrm{MHz})$ between the detected eigenfrequencies supports such interpretation but the elliptical shape of the free layer does not allow to conduct qualitative comparison of the model with the experiments. In the case of nanocontacts, there is no spatial confinement of the magnetic vortex motion in the film plane. The Oersted field of the current $^{51}$ forms a potential $W(\mathbf{X})$ proportional to $|\mathbf{X}|$ and the calculation approach should be modified.

\section{ACKNOWLEDGMENTS}

K.G. acknowledges support by IKERBASQUE (the Basque Science Foundation). This work was also supported by the Spanish Grants No. MAT2007-66719-C03-01 and No. CS2008-023.
${ }^{1}$ V. Novosad. F. Y. Fradin. P. E. Roy. K. Buchanan. K. Y. Guslienko. and S. D. Bader, Phys. Rev. B 72. 024455 (2005).

${ }^{2}$ I. Neudecker. K. Perzlmaier, F. Hoffmann. G. Woltersdorf. M. Buess, D. Weiss, and C. H. Back, Phys. Rev. B 73, 134426 (2006).

${ }^{3}$ F. G. Aliev, J. F. Sierra, A. A. Awad, G. N. Kakazei, D.-S. Han, S.-K. Kim, V. Metlushko, B. Ilic, and K. Y. Guslienko, Phys. Rev. B 79, 174433 (2009)

${ }^{4}$ J. P. Park, P. Eames, D. M. Engebretson, J. Berezovsky, and P. A. Crowell, Phys. Rev. B 67. 020403 (2003).

${ }^{5} \mathrm{M}$. Buess, R. Höllinger, T. Haug, K. Perzlmaier, U. Krey, D. Pescia, M. R. Scheinfein, D. Weiss, and C. H. Back, Phys. Rev. Lett. 93, 077207 (2004)

${ }^{6} \mathrm{X}$. Zhu, Z. Liu, V. Metlushko, P. Grutter, and M. R. Freeman, Plyys. Rev. B 71. 180408(R) (2005).

${ }^{7}$ S.-B. Choe, Y. Acreman. A. Scholl, A. Bauer, A. Doran. J. Stohr. and H. A. Padmore, Science 304, 420 (2004)

${ }^{8}$ A. Puzic, B. Van Waeyenberge. K. W. Chou, P. Fischer. H. Stoll. G. Schiitz, T. Tyliszczak, K. Rott, H. Brïckl, G. Reiss. I. Neudecker, T. Haug, M. Buess, and C. H. Back, J. Appl. Phys. 97, 10E704 (2005).

${ }^{9}$ K. Y. Guslienko, X. F. Han, D. J. Keavney, R. Divan, and S. D. Bader, Phys. Rev. Lett. 96, 067205 (2006).

${ }^{10}$ B. Van Waeyenberge, A. Puzic, H. Stoll, K. W. Chou, T. Tyliszczak, R. Hertel, M. Falmle, H. Brackl, K. Rott, G. Reiss, I. Neudecker, D. Weiss. C. H. Back. and G. Schatz. Nature (London) 444. 461 (2006).

${ }^{11}$ T. Shinjo. T. Okuno, R. Hassdorf, K. Shigeto, and T. Ono. Science 289. $930(2000)$.

${ }^{12}$ K. Y. Guslienko, V. Novosad, Y. Otani, H. Shima. and K. Fukamichi. Phys. Rev. B 65, 024414 (2001).

${ }^{13}$ R. P. Cowburn, D. K. Koltsov, A. O. Adeyeye, M. E. Welland, and D. M. Tricker, Phys. Rev. Lett. 83, 1042 (1999).

${ }^{14}$ A. Wachowiak, J. Wiebe, M. Bode, O. Pietzsch, M. Morgenstem, and R. Wiesendanger, Science 298, 577 (2002).

${ }^{15}$ A. A. Thiele, Phys. Rev. Lett. 30. 230 (1973).

${ }^{16}$ D. L. Huber. Phys. Rev. B 26, 3758 (1982).

${ }^{17}$ K. Yu. Guslienko. B. A. Ivanov. V. Novosad, H. Shima. Y. Otani. and K. Fukamichi, J. Appl. Phys. 91, 8037 (2002).

${ }^{18}$ K. Y. Guslienko. J. Nanosci. Nanotechn. 8. 2745 (2008).
${ }^{19}$ K. Y. Guslienko, A. N. Slavin. V. T. Tiberkevich, and S.-K. Kim, Phys. Rev. Lett. 101, 247203 (2008)

${ }^{20}$ K. Y. Guslienko. G. R. Aranda, and J. M. Gonzalez. Phys. Rev. B 81, 014414 (2010).

${ }^{21}$ K. Yamada, S. Kasai, Y. Nakatani, K. Kobayashi, H. Kohno, A. Thiaville, and T. Ono, Nature Mater. 6, 270 (2007).

${ }^{22}$ T. Okuno, K. Shigeto, T. Ono, K. Mibu, and T. Shinjo, J. Magn. Magn. Mater. 240, 1 (2002).

${ }^{23}$ R. Hertel. S. Gliga, M. Fälnnle. and C. M. Schneider, Plyys. Rev. Lett. 98. 117201 (2007).

${ }^{24}$ S. Choi, K.-S. Lee, K. Y. Guslienko, and S.-K. Kim, Phys. Rev. Lett. 98, 087205 (2007).

${ }^{25}$ K.-S. Lee, S.-K. Kim, Y.-S. Yu, Y.-S. Choi, K. Y. Guslienko, H. Jung, and P. Fischer, Phys. Rev. Lett. 101, 267206 (2008).

${ }^{26}$ L. F. Alvarez. O. Pla. and O. Chubykalo, Phys. Rev. B 61, 11613 (2000).

${ }^{27}$ G. Bertotti. C. Serpico. and I. D. Mayergoyz, Phys. Rev. Lett. 86. 724 (2001).

${ }^{28}$ R. W. Damon. Rev. Mod. Phys. 25, 239 (1953)

${ }^{29} \mathrm{~N}$. Bloembergen and S. Wang. Phys. Rev. 93, 72 (1954).

${ }^{30}$ G. V. Skrotskii and Yu. I. Alimov, Sov. Phys. JETP 35, 1035 (1959); 36, 899 (1959)

${ }^{31}$ H. Suhl, J. Phys. Chem. Solids 1, 209 (1957): J. Appl. Phys. 31. 935 (1960).

${ }^{32}$ M. Bater, O. Buttner, S. O. Demokritov, B. Hillebrands, V. Grimalsky. Yu. Rapoport. and A. N. Slavin. Phys. Rev. Lett. 81, 3769 (1998).

${ }^{3}$ J. Jorzick. S. O. Demokritov, B. Hillebrands, M. Bailleul, C. Fermon, K. Y. Guslienko. A. N. Slavin, D. V. Berkov, and N. L. Gom. Phys. Rev. Lett. 88. 047204 (2002); G. Gubbiotti, M. Conti, G. Carlotti, P. Candeloro, E. Di Fabrizio, K. Y. Guslienko, A. Andre, C. Bayer, and A. N. Slavin, J. Phys.: Condens. Matter 16, 7709 (2004).

${ }^{34}$ S. O. Demokritov, V. E. Demidov, O. Dzyapko, G. A. Melkov, A. A. Serga, B. Hillebrands, and A. N. Slavin, Nature (London) 443. 430 (2006)

${ }^{35}$ R. Yanes, R. Rozada, F, Garcia-Sanchez. O. Chubykalo-Fesenko, P. M. Pimentel. B. Leven. and B. Hillebrands. Phys. Rev. B 79, 224427 (2009).

${ }^{36}$ O. Chubykalo. J. D. Hannay, M. Wongsam. R. W. Chantrell, and 
J. M. Gonzalez, Phys. Rev. B 65, 184428 (2002).

${ }^{37}$ Yu. K. Fetisov and C. E. Patton, IEEE Trans. Magn. 40, 473 (2004).

${ }^{38}$ K. S. Buchanan. M. Grimsditch, F. Y. Fradin. S. D. Bader. and V. Novosad, Phys. Rev. Lett. 99, 267201 (2007).

${ }^{39}$ K. Y. Guslienko. Appl. Phys. Lett. 89, 022510 (2006).

${ }^{40}$ K. Y. Guslienko. W. Scholz. R. W. Chantrell. and V. Novosad. Phys. Rev. B 71. 144407 (2005).

${ }^{41}$ K.-S. Lee and S.-K. Kim, Appl. Phys. Lett. 91, 132511 (2007).

${ }^{42}$ K. S. Buchanan, P. E. Roy, M. Grimsditch, F. Y. Fradin, K. Y. Guslienko, S. D. Bader, and V. Novosad, Phys. Rev. B 74, 064404 (2006).

${ }^{43}$ Y.-S. Choi, S.-K. Kim, K.-S. Lee, and Y.-S. Yu, Appl. Phys. Lett. 93, 182508 (2008).

${ }^{41}$ I. S. Gradshteyn and I. M. Ryzhik. Table of Inegrals. Series and Products. 7th ed. (Academic Press, New York, 2007).
${ }^{45}$ X. M. Cheng, K. S. Buclanan, R. Divan, K. Y. Guslienko, and D. J. Keavney, Phys. Rev. B 79. 172411 (2009).

${ }^{46}$ A. Vansteenkiste, K. W. Chou, M. Weigand, M. Curcic, V. Sackmann, H. Stoll, T. Tyliszczak. G. Woltersdorf. C. H. Back, G. Schiitz. and B. Van Waeyenberge. Nat. Phys. 5, 332 (2009).

${ }^{47}$ K.-S. Lee. K. Y. Guslienko. J.-Y. Lee. and S.-K. Kim, Phys. Rev. B 76, 174410 (2007).

${ }^{48}$ A. Yamaguchi. K. Motoi. H. Miyajima, T. Sato, and Y. Nakatani, IEEE Trans. Magn. 46, 1994 (2010).

${ }^{49}$ A. V. Khvalkovskiy, J. Grollier, A. Dussaux, K. A. Zvezdin, and V. Cros, Phys. Rev. B 80, 140401 (2009).

${ }^{50}$ V. S. Pribiag, G. Finocchio, B. J. Williams, D. C. Ralph, and R. A. Buhrman, Phys. Rev. B 80, 180411 (2009).

${ }^{51}$ Q. Mistral, M. van Kampen, G. Hrkac, J.-V. Kim, T. Devolder, P. Crozat, C. Chappert, L. Lagae. and T. Schrefl, Phys. Rev. Lett. 100. 257201 (2008). 